\title{
The Interplay Between Genotypes and Family Relationships
} Reframing Concepts of Development and Prevention

\author{
David Reiss \\ Center for Family Research, Department of Psychiatry and Behavioral Sciences, George Washington University
}

\begin{abstract}
Children's genotypes and their social relationships are correlated throughout their development. Heritable characteristics of children evoke strong and specific responses from their parents; frequently, these same heritable characteristics also influence the children's adjustment. Moreover, parental heritable traits that influence their parenting are also transmitted to children and influence their children's adjustment. Thus, genetically influenced evocative processes from children and parental-transmission mechanisms influence the covariances between measures of family relationships and child development. These findings suggest new targets for preventing adverse development: altering parental responses to heritable characteristics of children and influencing the genetically influenced ontogeny of parenting.
\end{abstract}

KEYWORDS-genotype; relationships; parenting; prevention

Conventional models of psychological development acknowledge that genetic and social factors both play a role. Older models assumed that these two influences were independent from each other and that differences among individuals in personality development, cognitive development and psychological development could be explained by adding their effects together. More recently, it has become clear that, in many cases, the social environment interacts with genetic influences. For example, the genetic risk for schizophrenia seems to be fully expressed only when children at genetic risk grow up in families with high conflict, emotional restriction, and chaotic intergenerational boundaries (Tienari et al., 2004). Such a perspective still allows social and genetic variables to be thought of as relatively distinct: Genetic factors render individuals susceptible to adverse social

Address correspondence to David Reiss, Center for Family Research, Department of Psychiatry and Behavioral Sciences, George Washington University, $2300 \mathrm{~K}$ Street, NW, Washington, DC 20037; e-mail: cfrdxr@gwumc.edu. environments; then, at some point-perhaps in early childhood or much later in development-unfavorable social factors elicit behavioral difficulties.

Recent data suggest that genetic and social influences are even more intertwined, however. From early development through adulthood, genetic and social factors are correlated; that is, individuals' genotypes are associated with many specific characteristics of their environment. This association occurs in two ways. First, as can be inferred from twin, sibling, and adoption studies, heritable characteristics of children can evoke highly specific responses from the social environment. For example, certain heritable characteristics of children evoke warmth and involvement from their parents. More importantly, the same genetic factors that evoke parental warmth also contribute to a child's social responsibility, including adherence to community norms and helping and sharing behaviors. In the research of my colleagues and I, almost all of the covariance between maternal warmth and child social responsibility is due to these genetic influences common to both parenting and child development (Reiss, Neiderhiser, Hetherington, \& Plomin, 2000).

The second way such associations may occur is that heritable traits that influence a mother's or father's parenting may be genetically transmitted to their children. Those same traits in children may make them vulnerable to psychopathology. For example, a recent twin study suggests that heritable factors influence maternal smoking during pregnancy and, when transmitted to children, increase the childrens' likelihood of having conduct problems. These data raise questions about whether fetal exposure to tobacco products is the main cause of their postnatal conduct problems (Maughan, Taylor, Caspi, \& Moffitt, 2004).

In behavioral genetics, associations between individuals' genotypes and their environment are called genotype-environment correlations. When a correlation is due to the effects of heritable features stimulating responses from the environment, it is called an evocative genotype-environment correlation. When it is due to genes transmitted by parents to their children, the term is passive genotype-environment correlation. Use of the word 
genotype in this type of research signifies the cumulative effect of all genetic influences on a particular trait, as examined in studies that usually use twin, sibling, or adoption designs.

\section{GENOTYPE-ENVIRONMENT CORRELATIONS AND MECHANISMS OF DEVELOPMENT}

\section{Parent-Child Relationships May Amplify Genetic Influences}

Rowe (1981) first reported data suggesting evocative genotypeparenting correlations. Monozygotic (i.e. derived from a single egg and genetically identical) twins' reports of how accepted they were by their parents were correlated more than twice as highly as the reports of dizygotic (i.e. from different eggs and 50\% genetically related) twins. Figure 1 illustrates how monozygoticdizygotic comparisons are used to make inferences about such correlations. Rowe's finding was subsequently replicated many times using different methods of assessing parent-child relationships: interviews of parents (Goodman \& Stevenson, 1991), parental self-reports, and direct observation of parent-child relationships (O'Connor, Hetherington, Reiss, \& Plomin, 1995).
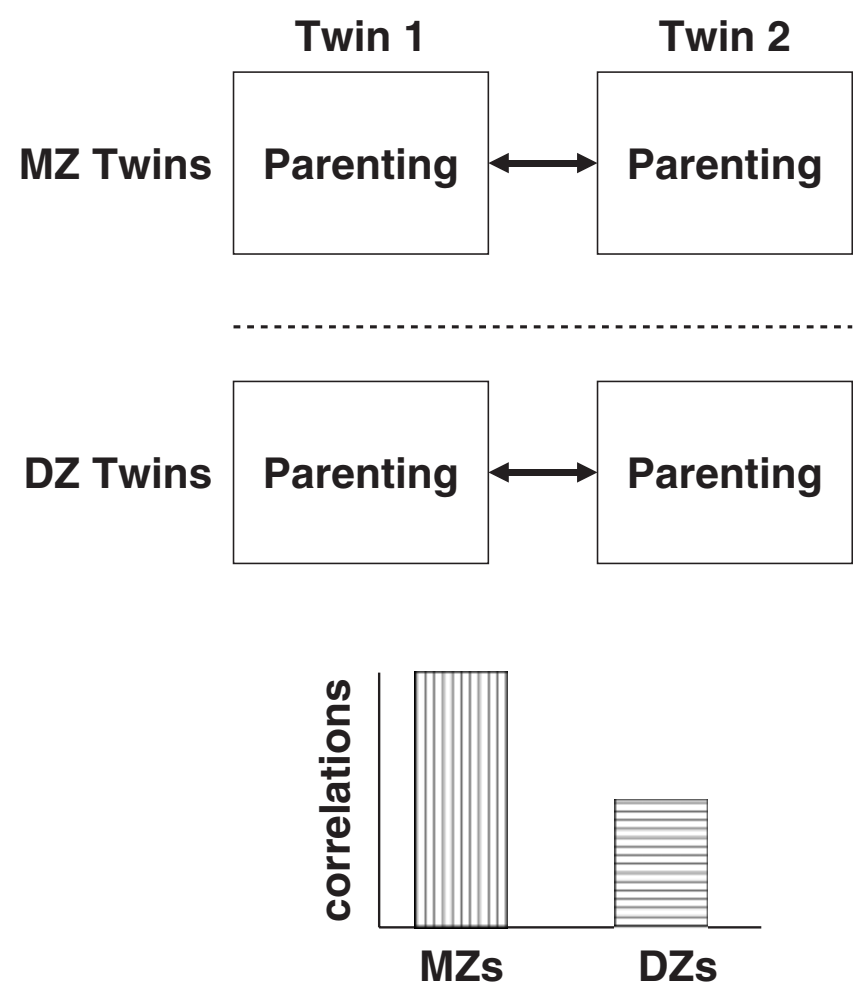

Fig. 1. Diagram showing how inferences about genetic influences on variation of a measured variable, in this case parenting, may be drawn from twin data. Boxes represent measured variable in a comparison of monozygotic (MZ) and dizygotic (DZ) child twins; the arrows represent correlations. The bar graph at the bottom of the figure represents example findings. The example finding shows MZ child twins correlate much more strongly than DZ child twins, enabling the inference that heritable characteristics of the child influence parenting (Reiss, Neiderhiser, Hetherington, \& Plomin, 2000).
These findings do not reflect parental bias due to their knowledge of whether their twins were monozygotic or dizygotic, since the findings also hold where monozygotic twins have been misdiagnosed as dizygotic (Goodman \& Stevenson, 1991).

Adoption studies have confirmed the importance of evocative genotype-parenting correlations: The behavior of an adoptive parent toward his or her child can be predicted from patterns of behavior in the birth parent. For example, two separate studies predicted adoptive parents' degree of harsh discipline and hostility toward their children from the level of aggressive behavior in the birth parents. These studies suggest that inherited externalizing (including aggressive and delinquent) behavior in the children evoked the response in the adoptive parents (Ge et al., 1996; O’Connor, Deater-Deckard, Fulker, Rutter, \& Plomin, 1998).

Heritable evoked parental responses have been reported from age 1 through late adolescence. For example, one study compared nonadoptive siblings, who share $50 \%$ of their individualdifferences genes, with siblings adopted from different birth parents. Data gathered at age 1 and again at age 2 suggested that children's genotypes greatly influenced how much intellectual stimulation their parents provided to them: Parental behavior correlated much higher toward the nonadoptive siblings than toward the adoptive siblings (Braungart, Plomin, Fulker, \& DeFries, 1992). Other studies have reported on genetic influences on parenting at age 3 , in middle childhood, and in adolescence. One longitudinal twin study suggested that heritable evocative effects increase across adolescence; this increase across age was particularly marked for fathers (Elkins, McGue, \& Iacono, 1997).

To study heritable evocative effects, the Nonshared Environment in Adolescent Development study (NEAD; Reiss et al., 2000 ) combined a twin design with a stepfamily design. We drew genetic inferences from comparisons among monozygotic twins, dizygotic twins, full siblings, half siblings (e.g., a mother brings a child from a previous marriage and has a child with her new husband) and unrelated siblings (i.e., each parent brings a child from a previous marriage). NEAD showed that heritable evocative effects may be quite specific. For example, genetic factors that evoke maternal warmth are distinct from those that evoke paternal warmth.

Additional findings reveal that heritable effects go beyond evocative effects on parents. The same genetic factors in a child that evoke particular parenting responses also influence many dimensions of their own adjustment during childhood and adolescence. Inferences about these influences are drawn by comparing cross correlations across sibling types (see Fig. 2). For example, a mother's harsh parenting towards sibling A can be correlated with the level of antisocial behavior of sibling B. Genetic influences on covariance are inferred when these cross correlations decline systematically from monozygotic twins at the highest to dizygotic twins and full siblings in the middle to unrelated siblings at the lowest.

NEAD found that over $70 \%$ of the covariance between a mother's hostile parenting and her adolescents' antisocial behavior was 

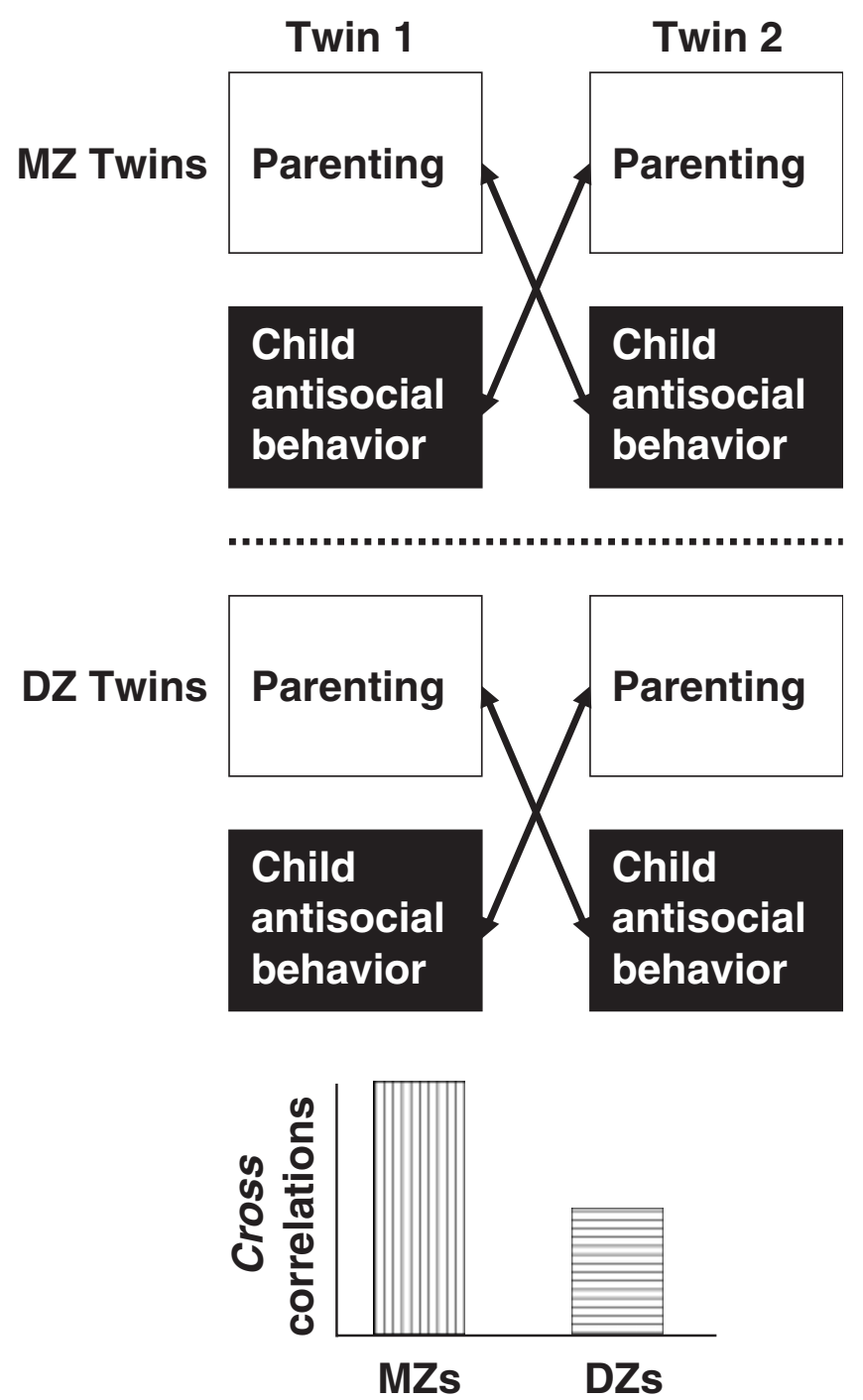

Fig. 2. Example cross correlation of parenting and child adjustment across sibling types. Parenting in one twin cross correlates with antisocial behavior in the other, more highly for monozygotic (MZ) twins than for dizygotic (DZ) twins. This suggests that the covariation between parenting and child antisocial behavior can be attributed to genetic influences common to both variables (Reiss et al., 2000).

accounted for by genetic influences common to both. These findings have been confirmed by several subsequent studies (e.g., Burt, Krueger, McGue, \& Iacono, 2003). NEAD found sizable genetic contributions to many other covariances including mothers' hostile parenting with impairment in adolescents' cognitive performance, fathers' warmth with adolescents'social responsibility, and fathers' hostility with adolescents' depression. NEAD, using longitudinal data collected from earlier and later adolescence, found that, in many cases, the child's heritable impact on parental response preceded the development of the behavior in question. For example, genetic influence on hostile parenting preceded the evolution of antisocial behavior.

Evocative genotype-parenting correlations may amplify more direct genetic influences on the child's problem behavior. In- deed, it is possible that parental responses to their children's heritable characteristics-responses to which the parents themselves are insensible-are critical for transforming heritable influences on children's temperaments into problems requiring clinical attention. To verify this hypothesis and test its significance for preventive intervention, my colleagues and I are currently conducting a prospective adoption study. We are following birth- and adoptive parents and adopted toddlers from age 9 months. This Early Growth and Development Study (EGADS) will allow us to pinpoint exactly what heritable noxious behaviors in the child evoke adverse parental responses and the consequence of these parental responses for subsequent child development.

\section{The Heritable Development of Parenting}

Evidence for passive genotype-parenting correlation requires evidence that (a) the parents' genes influence their parenting and (b) genetic factors that influence parenting are transmitted to their children and influence important dimensions of the children's adjustment. Evidence of this kind provides clues to childhood origins of parenting styles. For example, suppose it is observed that the same genetic factors that influence lack of warmth in mothers also influence depressive symptoms in their children. This would suggest that genetic factors link childhood internalizing with reduced maternal warmth, thereby offering clues about how genetically influenced parenting patterns unfold over the long term. Evidence for these passive effects comes from two sources.

First, studies using twins who are parents have shown genetic influences on dimensions of parenting. Our Twin Mom study investigated a sample of monozygotic and dizygotic twins who were mothers of adolescents. It showed that mothers' reports of their own warmth, hostility, and monitoring of the whereabouts of their children were more highly correlated for monozygotic than for dizygotic twins. A similar pattern of findings was shown using observer ratings for mothers' warmth and for children's ratings of their mothers' monitoring (Neiderhiser et al., 2004).

Second, adoption studies have found evidence for passive genetic links between parenting and the adjustment of children both in early childhood and in later adolescence. The correlation between parenting and child adjustment in birth families reflects evocative genotype-parenting correlations, environmental mechanisms, and passive genotype-parenting correlations. The last type are missing in adoptive families. Thus, by comparing correlations between parenting and child adjustment between the two groups it is possible to estimate-by elimination-the strength of passive genotype-parenting correlations. For example, one adoption study assessed parents' ratings of cohesiveness, lack of conflict, and open expression of feelings in their family during the time their children were 1 to 5 years old. For boys but not for girls, these ratings predicted teacher ratings of child delinquency and aggression at age 7 , but only for boys raised by 
their own birth parents (Braungart-Rieker, Rende, Plomin, \& DeFries, 1995). The correlations between parenting and teacherrated aggression were insignificant in adoptive families. The correlations between adolescent problems and ratings of the quality of family relationships by their mothers were higher in families in which parents reared their own children than in adoptive families (McGue, Sharma, \& Benson, 1996).

Taken together, these finding suggest that genotypic differences among parents influence their parenting and that these genotypic differences are transmitted to children, in whom they are manifested by psychiatric symptoms. We are currently investigating whether there are specific genetic links between childhood behavioral characteristics and patterns of parenting. For example, might internalizing problems in childhood be genetically linked to parental withdrawal and lack of support? Might childhood externalizing be genetically linked to aggressive and hostile parenting styles?

\section{Highlighting Relationship Influences}

Genetically informed studies highlight two sorts of family relationships that are linked with child psychological development independently of children's genotypes. Parent-child relationships are the first sort of such relationships identified by behavioral genetic data. For example, NEAD found that maternal rapport and affection was linked to adolescent autonomy and sociability. This is the case no matter what the child's genotype. Moreover, siblings within the same family are similar in their autonomy and sociability whether they are dizygotic or monozygotic twins or unrelated siblings. In contrast to parent hostility, the amount of maternal warmth received is also similar across both types of twins and unrelated siblings. Thus, taken together, our data suggest that mothers are relatively consistent in the positive feelings they show to children in their family and that all children benefit, no matter their genotype (Reiss et al., 2000).

Second, behavioral genetic data have highlighted nonparental family relationships that appear to influence childrens' development independently of their genotype. For example, NEAD showed that hostility and conflict in sibling relationships was strongly associated with adolescent antisocial behavior and depression. Conflict and hostility were highly reciprocal in adolescent siblings and put both siblings at equal risk for psychiatric symptoms independently of their genotypes. Moreover, NEAD showed a strong association between marital conflict and parentchild conflict on the one hand and sibling hostility on the other. More importantly, these links across family subsystems were independent of child genotype. Thus, in adolescence, hostility between siblings may be an indirect route through which family discord increases the vulnerability of children regardless of their genotype (Reiss et al., 2000).

Because it included the partners of the sisters who were the biological parents of the adolescent children, the Twin Mom study was able to yield valuable data on the role of adult genotypes in marital relationships. The study found that although genetic factors had a substantial influence on marital quality, as reported by both the twin siblings and their husbands, genetic factors explained little of the covariance between marital satisfaction and levels of wives' depressive symptoms. Rather, in this association, the dynamics of the marital relationships may play a central role (Spotts et al., 2004). These findings extend nongenetic studies of adult development that appeared to show that good marriages protect against depression and other behavior difficulties. However, nongenetic studies may miss heritable features of individuals that lead to both sustained, high-quality marriages and invulnerability to depression. Yet if the Twin Moms data is replicated, heritable features will seem unlikely to play a significant role in how marriages protect the marital partners.

\section{IMPLICATIONS: NEW TARGETS FOR PREVENTION}

Data on genotypes and family relationships offer three novel opportunities to design preventive interventions to forestall the development of serious problem behaviors and psychopathology.

First, findings suggesting that parent-child relationships amplify maladaptive genetic influences offer some of the most promising leads in preventing the expression of unfavorable genetic influences on many domains of child and adolescent adjustment. EGADS is designed to specify particular targets for intervention: parents' responses to heritable difficulties in their children. Numerous studies show that highly focused interventions can produce sustained changes in how parents respond to challenging children (Bakermans-Kranenburg, van Ijzendoorn, \& Juffer, 2003). EGADS is designed to ascertain whether such interventions might suppress the parental amplification process and thus diminish adverse genetic influences.

Second, findings on passive genotype correlations provide a new target for interventions: promoting favorable parenting. The discovery of genetic links between childhood behavior and parenting suggests some childhood and adolescent origins of parenting behavior that should be addressed in efforts to prevent risky parental behavior such as drug abuse during pregnancy or hostile and abusive parenting subsequently. For example, efforts to prevent the early emergence of conduct problems may prevent later serious antisocial behavior as well as abusive parenting.

Finally, studies of genotype-environment correlation suggest new psychosocial targets for preventing psychological and behavioral disorders: siblings and marriages. Techniques already developed for clinical interventions with maladaptive sibling relationships and with marriages might be refashioned for preventing psychological disorders in the siblings or marital partners.

\section{Recommended Reading}

Maughan, B., Taylor, A., Caspi, A., \& Moffitt, T.E. (2004). (See References)

Reiss, D., Pedersen, N.L., Cederblad, M., Lichtenstein, P., Hansson, K., Neiderhiser, J. M., et al. (2001). Genetic probes of three theories 
of maternal adjustment: I. Recent evidence and a model. Family Process, 40, 247-259.

Rutter, M., Pickles, A., Murray, R., \& Eaves, L. (2001). Testing hypotheses on specific environmental causal effects on behavior. Psychological Bulletin, 127, 291-324.

\section{REFERENCES}

Bakermans-Kranenburg, M.J., van Ijzendoorn, M.H., \& Juffer, F. (2003). Less is more: Meta-analyses of sensitivity and attachment interventions in early childhood. Psychological Bulletin, 129, 195-215.

Braungart, J.M., Plomin, R., Fulker, D.W., \& DeFries, J.C. (1992). Genetic mediation of the home environment during infancy: A sibling adoption study of the HOME. Developmental Psychology, 28, 1048-1055.

Braungart-Rieker, J., Rende, R.D., Plomin, R., \& DeFries, J.C. (1995). Genetic mediation of longitudinal associations between family environment and childhood behavior problems. Development \& Psychopathology, 7, 233-245.

Burt, S., Krueger, R.F., McGue, M., \& Iacono, W. (2003). Parent-child conflict and the comorbidity among childhood externalizing disorders. Archives of General Psychiatry, 60, 505-513.

Elkins, I.J., McGue, M., \& Iacono, W.G. (1997). Genetic and environmental influences on parent-son relationships: Evidence for increasing genetic influence during adolescence. Developmental Psychology, 33, 351-363.

Ge, X., Conger, R.D., Cadoret, R.J., Neiderhiser, J.M., Yates, W., Troughton, E., \& Stewart, M.A. (1996). The developmental interface between nature and nurture: A mutual influence model of child antisocial behavior and parent behaviors. Developmental Psychology, 32, 574-589.

Goodman, R., \& Stevenson, J. (1991). Parental criticism and warmth towards unrecognized monozygotic twins. Behavior and Brain Sciences, 14, 394-395.
Maughan, B., Taylor, A., Caspi, A., \& Moffitt, T.E. (2004). Prenatal smoking and early childhood conduct problems: Testing genetic and environmental explanations of the association. Archives of General Psychiatry, 61, 836-843.

McGue, M., Sharma, A., \& Benson, P. (1996). The effect of common rearing on adolescent adjustment: Evidence from a U.S. adoption cohort. Developmental Psychology, 32, 604-613.

Neiderhiser, J.M., Reiss, D., Pedersen, N.L., Lichtenstein, P., Spotts, E.L., Hansson, K., Cederblad, M., \& Elthammar, O. (2004). Genetic and environmental influences on mothering of adolescents: A comparison of two samples. Developmental Psychology, 40, $335-351$.

O'Connor, T.G., Deater-Deckard, K., Fulker, D., Rutter, M., \& Plomin, R. (1998). Genotype-environment correlations in late childhood and early adolescence: Antisocial behavioral problems and coercive parenting. Developmental Psychology, 34, 970-981.

O'Connor, T.G., Hetherington, E.M., Reiss, D., \& Plomin, R. (1995). A twin-sibling study of observed parent-adolescent interactions. Child Development, 66, 812-829.

Reiss, D., Neiderhiser, J., Hetherington, E.M., \& Plomin, R. (2000). The relationship code: Deciphering genetic and social patterns in adolescent development. Cambridge, MA: Harvard University Press.

Rowe, D.C. (1981). Environmental and genetic influences on dimensions of perceived parenting: A twin study. Developmental Psychology, 17, 203-208.

Spotts, E.L., Neiderhiser, J.M., Ganiban, J., Reiss, D., Lichtenstein, P., Hansson, K., Cederblad, M., \& Pedersen, N. (2004). Accounting for depressive symptoms in women: A twin study of associations with interpersonal relationships. Journal of Affective Disorders, 82, 101-111.

Tienari, P., Wynne, L.C., Sorri, A., Lahti, I., Laksy, K., Moring, J., Naarala, M., Nieminen, P., \& Wahlberg, K. (2004). Genotype-environment interaction in schizophrenia spectrum disorder. British Journal of Psychiatry, 184, 216-222. 